\title{
The Current Consensus on the Evaluation and Management of Antenatal Hydronephrosis
}

\section{Pradyumna Pan*}

Pediatric Surgery Unit, Ashish Hospital and Research Centre, Jabalpur,Madhya Pradesh, India

*Corresponding Author: Pradyumna Pan, Pediatric Surgery Unit, Ashish Hospital and Research Centre, Jabalpur,Madhya Pradesh, India.

Received: July 29, 2019; Published: August 13, 2019

\section{Abstract}

The antenatal screening has brought about an increased detection of anomalies of the kidneys and urinary tract. All patients with antenatal hydronephrosis should undergo postnatal ultrasonography; the intensity of subsequent evaluation depends on the anterior pelvic diameter of the renal pelvis and Society for Fetal Urology grading. This article is composed of a plan to give thorough data concerning the postnatal management of antenatally detected hydronephrosis.

Keywords: Antenatal Hydronephrosis; Prenatal Diagnosis; Vesicoureteral Reflux; Ureteropelvic Junction Obstruction

\section{Defining and grading ANH}

The detection of renal abnormalities during antenatal ultrasonography was first reported by Garrett et al, in 1970 [1]. With the advent of antenatal sonography, detection of fetal hydronephrosis is more frequent in recent times. Antenatal hydronephrosis affects $1 \%$ to $5 \%$ of all pregnancies and is one of the most commonly detected anomalies [2]. Antenatal diagnosis is crucial as it emphasizes the fetus at risk and facilitates parental counselling. The condition is bilateral in $17-54 \%$ [3].

Antenatal hydronephrosis (ANH) was defined based on the grading system of the Society for Fetal Urology and APD measuring system. Renal pelvis dilation greater than $4 \mathrm{~mm}$ in less than 33 weeks or greater than $7 \mathrm{~mm}$ in a gestational age more than 33 weeks is taken as fetal hydronephrosis [4].

\section{Etiology of ANH}

The commonest cause of ANH is transient/ physiologic, which is seen in $50-70 \%$. Next commonly seen are ureteropelvic junction obstruction (10-30\%) and vesicoureteral reflux (10-30\%). Other causes are ureterovesical junction obstruction seen in 5-15\%, multicystic dysplastic kidney in 1-5\%, posterior urethral valves (15\%) and ureterocele (1-3\%) [5].

\section{Antenatal counseling}

The parents are counselled, and all their queries are to be answered. Addressing parental anxiety and concerns is as important as the clinical management of the child. This timely recognition helps in improving postnatal results and assists in preserving the renal function. If not detected by antenatal ultrasonography and subsequently managed, many of these anomalies would manifest later in life with complications. Antenatal identification of urinary tract dilatation does not necessarily indicate an obstruction, nor give any functional suggestion of an affected kidney [6].

\section{Prenatal evaluation}

Antenatal hydronephrosis detected at 16-20 weeks' gestation should include evaluation for lower urinary tract obstruction, renal dysplasia, and extra renal structural malformations. In fetuses, with unilateral hydronephrosis at least one follow-up ultrasound be performed in the third trimester and fetuses with bilateral hydronephrosis be monitored frequently. The frequency of monitoring varies from 4 to 6 weeks, depending on gestation at which ANH was detected, its severity and presence of oligohydramnios [4].

\section{Prenatal intervention}

The goal of fetal intervention would be to relieve the obstruction and allow for normal renal development, maintain the amniotic fluid levels and to allow for normal lung development. Studies have shown that urinary obstruction can cause renal dysplasia and relief of that obstruction can prevent if performed early enough [7]. The diagnostic and therapeutic interventions are to be considered for fetuses with lower urinary tract obstruction and oligohydramnios only at specialized centers. Termination of pregnancy is not recommended in fetuses with unilateral or bilateral ANH, except in the presence of extra renal life threatening abnormality. The bene- 
fits of such intervention, usually performed during the mid-second trimester, are equivocal. There is no evidence that this intervention improves long term renal outcome or reduces mortality in fetuses with less severe disease $[8,9]$. The procedure is associated with significant fetal and maternal morbidity and fetal mortality [10].

\section{Postnatal management}

The antenatal hydronephrosis is classified based on renal pelvic antero posterior diameter. Mild is less than $5 \mathrm{~mm}$, moderate 5-10 $\mathrm{mm}$ and severe is $>15 \mathrm{~mm}$ [11]. The grading system of Society for Fetal Urology for postnatal $\mathrm{HN}$ is based on ultrasound findings of the degree of renal-pelvic and calyceal dilation and takes into account the integrity of the parenchyma. At Grade 0, there is no HN. At Grade 1, the renal pelvis only is visualized with slight separation of the central renal echo complex. In Grade 2 the renal pelvis is further dilated and a single or a few but not all calices are identified in addition to the renal pelvis. In Grade 3, the renal pelvis is dilated and there are fluid filled calices throughout the kidney, but the renal parenchyma is of normal thickness. Grade 4 have a similar appearance of the calices as grade 3 , but there is parenchymal thinning over the calices [12].

\section{Immediate evaluation}

All newborn with history of ANH should have postnatal ultrasound examination at the end of the first week of life (3-7days) before the hospital discharge. The postnatal ultasound should include evaluation for calyceal dilation, cortical cysts and renal echogenicity, ureteric dilation, ureterocele, bladder wall abnormalities and bladder emptying.

In suspected lower urinary tract obstruction, oligohydramnios or severe ANH ultrasound should be performed within 24-48 hr of birth. Infants with lower urinary tract obstruction should be immediately attended for appropriate intervention.

\section{How to follow up?}

The critical questions to be answered during follow up of these infants are: (1) Do they need prophylactic antibiotics; (2) Do they need VCUG and (3) When to repeat ultrasound?

Management of infants diagnosed with isolated ANH and an abnormal initial postnatal pelvicalyceal dilatation.

Neonates with abnormal ultrasound examination in the first week of life should undergo a repeat examination at 4-6 weeks.

\section{The risk stratification}

Low risk

Patients with SFU grade 1 or $2 \mathrm{HN}$ or postnatal APDs $<10 \mathrm{~mm}$
Intermediate risk

Patients with SFU grade 3 or bilateral HN or postnatal APDs 10$20 \mathrm{~mm}$

\section{High risk}

Patients with SFU grade $4 \mathrm{HN}$ or postnatal APDs $>20 \mathrm{~mm}$ [13]

The presence of two normal postnatal renal ultrasounds excludes the presence of significant renal disease including VUR. Diuretic renograms are taken to decide if urinary tract obstruction is present in patients with persistent hydronephrosis in the absence of VUR. Infants with moderate to severe unilateral or bilateral hydronephrosis (SFU grade 3-4 or APD $>10 \mathrm{~mm}$ ) and dilated ureter(s) who do not show VUR should undergo diuretic renography [4].

\section{Evaluation for VUR in children with isolated ANH}

Vesico ureteral reflux occurs in $10-20 \%$ of patients with antenatal hydronephrosis and is associated with substantial morbidity [14]. Many authors recommend performing an MCUG to all children with ANH irrespective of the degree of postnatal dilatation in view of negligible risks related to MCUG in the neonatal period and the hazard associated with VUR and UTI even in mild ANH $[14,15]$.

MCUG is to be performed in patients with unilateral or bilateral hydronephrosis with renal pelvic APD $>10 \mathrm{~mm}$, SFU grade 2, 3, 4 and with ureteric dilatation. MCUG be carried out early, within 24$72 \mathrm{~h}$ of life, in patients with suspected lower urinary tract obstruction. In other cases, the procedure is to be performed at 4-6 weeks of age [13].

\section{Surgical intervention}

Indications for surgical intervention are to be considered in posterior urethral valves immediately, non-response conservative therapy, deterioration of renal function, progressive dilation in patients with bilateral hydronephrosis, and recurrent pyelonephritis $[13,16]$.

\section{The role of antibiotic prophylaxis in infants with ANH}

The decision to place all children with ANH on prophylactic antibiotics is controversial. The ultimate goal is the prevention of febrile UTI and its attendant risk of renal damage. Many authors reported a lower frequency of UTI and higher spontaneous resolution rate in patients with low-grade $\mathrm{HN}$ as compared to patients with high grade [17]. Coelho., et al. reported female gender and presence of uropathy as independent predictors of UTI in patients with AHN [18]. 
Bilateral hydronephrosis

Infants with bilateral hydronephrosis are at an increased risk of infection and renal function deterioration as compared to children with unilateral hydronephrosis [19]. The differential renal function on renogram is not predictable for the need for surgery. The following guidelines to manage this group of patients are; if APD decreases by $>10 \%$ in the prone position in any renal unit then USG monthly for six months and three monthly till two years. VCUG is to be done at 4-6 weeks. No decrease in APD in any renal unit or increase in APD, VCUG and renogram are to be done followed by surgical intervention.

\section{Conclusion}

The level of ANH can be utilized as a guide for deciding about diagnostic imaging and treatment. Recent results strongly submit that fetal hydronephrosis less than $5 \mathrm{~mm}$, SFU grade 1-2 runs a benign course, needs minimal investigation and the likelihood of spontaneous resolution is high. In APD greater than $15 \mathrm{~mm}$, SFU grades 3-4, bilateral disease, or bladder distension thorough postnatal evaluation and regular follow-up is necessitated to plan a timely intervention. It is considered that MCUG is not obligatory in asymptomatic newborns with mild ANH.

\section{Competing Interests}

The author declare that they have no competing interests.

\section{Funding}

Not applicable.

\section{Bibliography}

1. Garrett WJ., et al. "Prenatal diagnosis of fetal polycystic kidney by ultrasound". Australian and New Zealand Journal of Obstetrics and Gynaecology 10.1 (1970): 7-9.

2. Blyth B., et al. "Antenatal diagnosis and subsequent management of hydronephrosis". Journal of Urology (1993): 693-698.

3. Broadley P., et al. "The 4-year outcome following the demonstration of bilateral renal pelvic dilatation on prenatal renal ultrasound". The British Journal of Radiology 72.855 (1999): 265-270.

4. Sinha A., et al. "Revised Guidelines on Management of Antenatal Hydronephrosis". Indian Pediatrics 50 (2013): 215-231.

5. Nguyen HT., et al. "The Society for Fetal Urology consensus statement on the evaluation and management of antenatal hydronephrosis". Journal of Pediatric Urology 6.3 (2010): 212231.

6. Valent-Moric B., et al. "Postnatal evaluation and outcome of infants with antenatal hydronephrosis". Acta Clinica Croatica 50.4 (2011): 451-455.
7. Herndon CD., et al. "Consensus on the prenatal management of antenatally detected urological abnormalities". Journal of Urology 164.3-2 (2000): 1052-1056.

8. McLorie G., et al. "Outcome analysis of vesico amniotic shunting in a comprehensive population". Journal of Urology 166.3 (2001): 1036-1040.

9. Biard JM., et al. "Long-term outcomes in children treated by prenatal vesicoamniotic shunting for lower urinary tract obstruction". Obstetrics and Gynecology 106.3 (2005): 503-508.

10. Sharma G and Sharma A. "Postnatal management of antenatally detected hydronephrosis". World Journal of Clinical Urology 3.3 (2014): 283-294.

11. Dhillon HK. "Prenatally diagnosed hydronephrosis: the Great Ormond Street experience". British Journal of Urology 81.2 (1998): 39-44.

12. Nguyen HT., et al. "The Society for Fetal Urology consensus statement on the evaluation and management of antenatal hydronephrosis". Journal of Pediatric Urology 6.3 (2010): 212231.

13. Pan P. "The fate of antenatal renal pelvis dilatation: a prospective postnatal cohort study". International Journal of Contemporary Pediatrics 6.4 (2019):1646-1651.

14. Carlos R and Estrada Jr CR. "Prenatal hydronephrosis: early evaluation". Current Opinion in Urology 18.4 (2008): 401-403.

15. Vates TS., et al. "Complications of voiding cysto urethrography in the evaluation of infants with prenatally detected hydronephrosis". Journal of Urology 162.3-2 (1999):1221-1223.

16. Sidhu G., et al. "Outcome of isolated antenatal hydronephrosis: a systematic review and meta-analysis". Pediatric Nephrology 21.2 (2006): 218-224.

17. Gokaslan F., et al. "Evaluation and Outcome of Antenatal Hydronephrosis: A Prospective Study". Renal Failure 34.6 (2012): 718-721.

18. Coelho GM., et al. "Outcome of isolated antenatal hydronephrosis: A prospective cohort study". Pediatric Nephrology 22.10 (2007):1727-1734.

19. Davenport MT., et al. "Antenatally diagnosed hydronephrosis: current postnatal management". Pediatric Surgery International 29.3 (2013):207-214.

Volume 2 Issue 9 September 2019 (C) All rights are reserved by Pradyumna Pan. 